\title{
Increased Expression of Oct4, Nanog and CD24 Predicts Poor Response to Chemo-Radiotherapy and Unfavourable Prognosis in Locally Advanced Oral Squamous Cell Carcinoma
}

\author{
Sridhar Mishra', Vandana Tiwari', Aditi Arora', Seema Gupta², Nidhi Anand², \\ Nuzhat Husain ${ }^{1 *}$
}

\begin{abstract}
Background: Current study investigates the role of Oct4, Nanog and CD24 in locally-advanced oral squamous cell carcinoma (OSCC), to evaluate whether the expression of these markers can predict efficacy of neoadjuvantchemo-radiotherapy and survival of patients. Methods: Biomarker expression was evaluated in 50 homogenously treated patients of locally-advanced OSCC. Results: Clinical response was complete in 30\% ( $\mathrm{n}=15)$, partial response in $46 \%(n=23)$, no response in $24 \%(n=12)$. Pathologically, $74 \%$ patents $(n=37)$ were responders and $26 \%$ were non-responders $(\mathrm{n}=13)$. Biomarker-overexpression was seen in $46 \%$ cases for Oct $4,54 \%$ cases for Nanog and $58 \%$ cases for CD24. Oct4, Nanog and CD24 expression showed significant correlation with clinical and pathological response $(p<0.05)$. Three year recurrence-free survival was $71 \%$, overall survival was $66 \%$. Post-treatment advanced pathological N (ypN), post treatment advanced pathological TNM (ypTNM) stage, clinical non-response, pathologic non-response, positive/high expression of all three biomarkers had a significant negative impact on recurrence-free and overall survival. Conclusions: Expressions of Oct4, Nanog and CD24 have significant association with treatment response and survival in patients with locally advanced OSCC treated with neoadjuvant chemo-radiation. Survival of these patients is significantly affected by ypN stage, ypTNM stage, expression of all three biomarkers, clinical and pathological response to neoadjuvant therapy.
\end{abstract}

Keywords: Oral squamous cell carcinoma- Oct4- Nanog-CD24- cancer stem cells

Asian Pac J Cancer Prev, 21 (9), 2539-2547

\section{Introduction}

Oral Squamous cell carcinoma (OSCC) is a common malignancy in the oral cavity. Advances in the field of reconstructive surgery and diagnostic modalities had led to improvement in the survival of patients with OSCC. Local recurrence and distant metastasis occur. Unfortunately, advanced OSCC is refractory to treatment and leads to death in $>50 \%$ of the cases (Jemal et al., 2011). Neoadjuvant chemo-radiotherapy is now an established modality for management of loco regional disease in patients with locally advanced operable OSCC (Kirita et al., 2012). Neoadjuvant chemo-radiotherapy helps in down-staging the primary tumor, making it resectable and eliminating micro metastases. Kirita et al., (2012), showed that preoperative cisplatin based chemotherapy and concurrent radiotherapy led to a clinical tumor response in $92.8 \%$ patients, with an overall 5-year survival of $79.3 \%$ in locally advanced resectable OSCC. However, all patients with advanced OSCC do not satisfactorily respond to neoadjuvant chemo-radiotherapy. It is hence critical to evaluate the molecular mechanisms for differential sensitivity to chemo-radiotherapy and detect molecular markers that can predict response.

It has been observed that cells that display stem cell-like characteristics within the tumor possess significant resistance to the current treatment modalities and promote tumor recurrence (Guo et al., 2014; Yanamoto et al., 2014). Obstinate properties of cancer stem cells (CSCs) against conventional chemotherapy regimens could explain anti-cancer therapy failure and recurrence in a number of cancer patients including those of OSCC in whom poor prognosis is related to the low response to chemotherapeutic drugs. Epithelial to mesenchymal transition (EMT) is a genetic hallmark of CSCs; change in tumor microenvironment stimulates EMT process that induces invasion and metastasis of tumors during cancer progression (Gupta et al., 2006). Thus understanding the role of CSCs in tumor initiation and progression has become a major focus in stem cell biology and cancer research.

Oct4 (Pou5f1) is a transcription factor, which is 
strongly expressed in undifferentiated stem cells, which maintains pluripotency (Nichols et al., 1998; Pesce et al., 1998; Mitsui et al., 2003). Nanog, a downstream target of Oct 4 , is a homeodomain-containing protein which plays a key role in the maintenance, self-renewal and pluripotency of embryonic stem cells (Pesce et al., 1998; Mitsui et al., 2003). Inappropriate and untimely activation of Oct4 and Nanog result in CSCs instead of differentiated somatic or normal pluripotent stem cells. CD24 biomarker expression is associated with aggressive tumours showing increase proliferative activity and invasion (Koukourakis et al., 2012; Kwon et al., 2007). CD24 is expressed in a variety of cancers and has been associated with shorter patient survival rates (Kristiansen et al., 2003a; 2003b; Sung et al., 2010; Choi et al., 2007; Chou et al., 2007; Sano et al., 2009)

In the current study, we analyzed the expression of and patterns of expression of Oct4, Nanog and CD24 in a series of patients with locally advanced OSCC undergoing neoadjuvant chemo-radiotherapy, with the aim to evaluate whether the expression of these CSC markers correlate with efficacy of neoadjuvant chemo-radiotherapy response and survival in patients with OSCC.

\section{Materials and Methods}

Patients: This study was conducted in the Department of Pathology, Dr. Ram Manohar Lohia Institute of Medical Sciences, Lucknow. Fifty patients of locally advanced OSCC, who were treated with neoadjuvant chemoradiotherapy followed by surgical resection, were enrolled from a retrospective series. The inclusion criteria were (i) availability of a diagnostic biopsy in treatment naïve cases of OSCC, (ii) clinical stage III-IVa/b with no evidence of distant metastatic disease (M0), (iii) Patients who received completed regime of neoadjuvant chemo-radiotherapy and underwent surgical resection with curative intent, (iv) no other treatment given. The data of all patients was collected from medical records. Location of primary tumour and demographics of patients were recorded in all patients. The patients were staged clinically in preoperative phase and in pathological specimen postoperatively. retrospectively according to TNM classification proposed by American Joint Committee on Cancer (AJCC) (Amin and Edge 2017). The tumor grade was assigned according to World Health Organization classification (Pindborg et al., 2012) newer version available

Treatment Protocol: All patients received chemoradiotherapy by external beam conventional method (200cGy/fraction/day for 5 days a week) to a total dose of $70 \mathrm{~Gy}$ in 35 fractions in 7-weeks to primary tumor site and neck along with concurrent weekly cisplatin (40mg/m²) (Gupta et al., 2009). Cisplatin was administered ambulatory with $1 \mathrm{~L}$ intravenous hydration along with adequate antiemetic prophylaxis. Surgical resection was performed 4-6 weeks after the last dose of chemo-radiation and comprised of resection of primary tumour site with a margin of at least $1 \mathrm{~cm}$ along with neck node dissection (supraomohyoid neck dissection for clinically node negative and modified radical neck dissection for clinically positive nodes) as described by the
American Head and Neck society (Robbins et al., 2002).

Assessment of clinical response to neoadjuvant chemo-radiotherapy: The Response Evaluation Criteria in Solid Tumors (RECIST), version 1.1, was used to define objective soft-tissue response (Eisenhauer et al., 2009). Response to treatment of measurable lesions was assessed with contrast-enhanced computed tomography (CECT) scan 6 weeks after neoadjuvant chemo-radiotherapy.

- Complete Response (CR): “disappearance of all target lesions with any pathological lymph nodes must have reduction in short axis to $<10 \mathrm{~mm}$."

- Partial Response (PR): “At least a 30\% decrease in the sum of diameters of target lesions, taking as reference the baseline sum diameters."

- Progressive Disease (PD): “At least a 20\% increase in the sum of diameters of target lesions, taking as reference the smallest sum on study. In addition to the relative increase of $20 \%$, the sum must also demonstrate an absolute increase of at least $5 \mathrm{~mm}$."

- Stable Disease (SD): "Neither sufficient shrinkage to qualify for PR nor sufficient increase to qualify for $\mathrm{PD}$, taking as reference the smallest sum diameters while on study."

Clinical response was categorized as complete responders, partial responders and non-responders. Non responders included patients with progressive disease.

Assessment of pathologic response: It was evaluated on resection specimen using 4 grade of pathological regression (Braun et al., 1989)

- Regression grade 1 (RG1 or complete pathologic response): No residual viable tumour

- Regression grade 2 (RG2 or microscopic residual foci): less than $5 \%$ viable tumour

- Regression grade 3 (RG3): 5-50\% viable tumour

- Regression grade 4 (RG3): more than 50\% viable tumour

RG1 and RG2 were categorized as responders, while RG3 and RG4 as non-responders.

Immunohistochemistry: Formalin fixed paraffin embedded tumour blocks of pre-treatment biopsy sample of patients were used for Immuohistochemical (IHC) analysis for this study.Tissue sections of $5 \mu \mathrm{m}$ were deparaffinised in xylene and then re-hydrated with sequential washes of ethanol. Endogenous peroxidase activity was inhibited with $3 \%$ hydrogen peroxidase (Loba Chemie, India) in methanol for 30 minute. For antigen retrieval, slides were placed in $50 \mathrm{ml}$ citrate buffer pH6.0 to unmask the epitopes. Tissue sections were then incubated with various antibodies. Anti-human antibodies Oct4 (Sigma, USA) used at a dilution of 1:100; Nanog (Thermo Scientific, USA) at a dilution of 1:75, and CD24 (Thermo Scientific, USA) in dilution of 1:25, were added for incubation followed by washing with wash buffer, three times followed by treatment with polymer based secondary antibody kit with 3'3 diaminobenzidine tetra hydrochloride (DAB), as substrate (DAKO, Denmark). All sections were counterstained with $0.1 \%$ haematoxylin and fixed with permanent mounting medium and covered with glass cover slips. Negative controls and recommended positive controls were used. The expression of stem cell markers was assessed as a percentage of positive tumours cells in 
hot spots (10 High Power Fields) as reported previously (Soni et al., 2014). Two pathologists (N.H, A.A) scored all samples blindly without knowing clinical characteristics and prognosis.

Evaluation of staining for Oct-4, Nanog and CD24: For evaluating expression of both Oct4 and Nanog only nuclear staining was considered as positive. The membranous and the cytoplasmic staining of CD24 was evaluated separately, and cytoplasmic staining was considered positive. IHC results were assessed in terms of the proportion of tumor cell staining according to the previous published method with modifications.23 For statistical analyses, $<10 \%$ expression was consider as negative and $\geq 10 \%$ as positive. Figure 1 shows representative examples of Oct4, Nanog and CD24 immunohistochemistry.

Outcomes: Variables assessed were age, sex, risk factors (including tobacco consumption, betel nut chewing, human papilloma virus infection, and alcohol consumption), location of tumor, grade of tumor, clinical $\mathrm{N}$ and TNM stage, pathologic $\mathrm{N}(\mathrm{ypN})$ and TNM stage (ypTNM) after neoadjuvant treatment post-treatment pathologic and clinical response, as well as the expression of Oct4, Nanog and CD24. Overall survival was the primary end point of our study. Secondary end points were response to treatment and recurrence-free survival. The time from surgery to death due to any cause was defined as overall survival. The time from surgery to recurrence of cancer (local or distant) or death without recurrence was defined as recurrence-free survival.

Statistical Analysis: Statistical analysis was performed using the Statistical Package for Social Sciences (SPSS) analysis software version 20 . The association of clinical and pathological response after neoadjuvant therapy with expression of biomarkers and clinico-pathological parameters was assessed using either Chi-square $\left(\chi^{2}\right)$ methodor Fisher's exact tests, as appropriate. Association of mean expression of biomarker with clinico-pathological parameters and type of response was assessed by Mann-Whitney U test; one way analysis of variance (ANOVA) and Kruskal Wallis test (for more than two groups, as appropriate). Cox proportional hazards regression analysis was performed to evaluate the effects of biomarkers and other clinico-pathological variables on recurrence free survival and overall survival. Statistical significance was defined as $\mathrm{p}$-value $<0.05$.

\section{Results}

Characteristics of the patients: A total of 50 cases were enrolled in the study, which included $82 \%$ males $(n=41)$ and $18 \%$ females $(n=9)$ with age ranging from 25 to 75 years $(49.3 \pm 11.3$ years $)$. The location of primary tumour was buccal mucosa in $42 \%(n=21)$, tongue in $28 \%(n=14)$, retro molar trigone in $10 \%(\mathrm{n}=5)$, and other (cheek, palate and lip) in $20 \%(\mathrm{n}=10)$. The baseline demographics and clinicopathological parameters have been summarised in Table 1. Clinical N stage was N0 in $22 \%(n=11)$, and

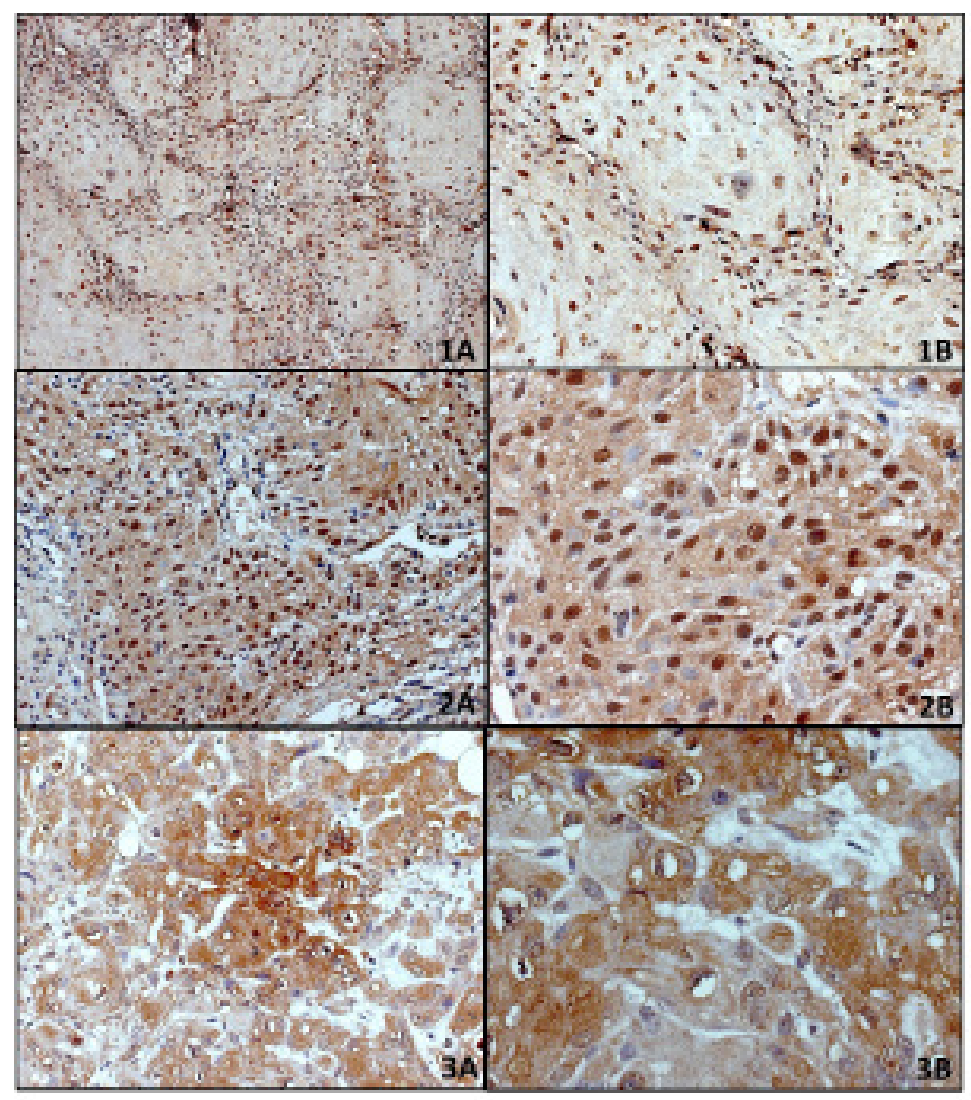

Figure 1. Micro Photograph Showing Immuohistochemical Staining for Oct4, Nanog and CD24 Expressions in Oral Squamous Cell Carcinoma. High positive expression of Oct4 in the nucleus of tumor cells (1a) 20X; (1b) 40X. High positive expression of Nanog in the nucleus of tumor cells (2a) 20X; (2b) 40X. High positive expressions of CD24 in the cytoplasm of tumor cells (3a) 20X; (3b) 40X. (DAB x 125 x digital magnification). 
$\mathrm{N}+$ in $78 \%(\mathrm{n}=39)$. Clinical TNM stage was III in $44 \%$ $(\mathrm{n}=22)$, and $\mathrm{IVa} / \mathrm{b}$ in $56 \%(\mathrm{n}=28)$. Pathological $\mathrm{N}$ stage after neoadjuvant therapy (ypN stage) was 0 in $70 \%$ $(n=35), 1$ in $24 \%(n=12)$ and 3 in $6 \%(n=3)$. Pathological TNM stage after neoadjuvant therapy (ypTNM stage) was $0: 1: 2: 3: 4$ in $40 \%(n=20): 18 \%(n=9): 12 \%(n=6): 16 \%$ $(n=8): 14 \%(n=7)$ respectively.

Out of 50 patients, clinical response was complete in $30 \%(\mathrm{n}=15)$, partial response in $46 \%(\mathrm{n}=23)$, no response in $24 \%$ (stable disease in 7 and progressive disease in 5). Pathologically, $74 \%$ patents $(n=37)$ were responders, and $26 \%$ were non-responders $(n=13)$. Fifteen patients $(30 \%)$ were classified as RG1, 22 (44\%) as RG2, 7 (14\%) as RG3 and $6(12 \%)$ as RG4. Follow-up data were available for all 50 patients. At follow-up of 36 months, 17 patients (34\%) had died: 10 owing to tumor recurrence and 7 owing to other causes. Of the 33 surviving patients $(66 \%)$, two were alive with tumor recurrence, and 31 were free of tumor. Of all 50 patients, 12 (16\%) developed tumor recurrence (8 local, 2 regional, and 2 distant metastasis).

Association of clinical and pathological response with qualitative biomarker expression and clinico-pathologic parameters: Based on the chosen cut off levels ( $>10 \%)$, over expression was seen in 23 of $50(46 \%)$ cases for Oct4, in 27 (54\%) for Nanog and in $29(58 \%)$ for CD24. A statistically significant association was found between negative expression $(<10 \%)$ of Oct4, Nanog, CD24 and clinical response $\left(\chi^{2}=13.28\right.$, p value $=0.001$ for Oct $4, \chi^{2}$ $=15.30, \mathrm{p}$ value $=0.0001$ for Nanog and $\chi^{2}=7.93$, $\mathrm{p}$ value $=0.01$ for CD24). Similarly, a statistically significant association was found between negative expression $(<10 \%)$ of Oct4, Nanog, CD24 and pathological response $\left(\chi^{2}=8.55, \mathrm{p}\right.$ value $=0.003$ for Oct $4, \chi^{2}=5.07, \mathrm{p}$ value $=$ 0.02 for Nanog and $\chi^{2}=2.38$, $\mathrm{p}$ value $=0.03$ for CD24). The relationship between biomarker expression and response has been summarised in Table 2.

Association of quantitative expression of biomarkers with demographic characteristics and response to neoadjuvant therapy: Significant difference was observed in the mean expression of all three biomarkers among pathological responders and non-responders (17.32 \pm 6.83 vs. $37.15 \pm 13.65$ for Oct $4 ; 18.86 \pm 10.21$ vs. $39.92 \pm 13.93$ for Nanog; $22.37 \pm 12.26$ vs. $47.93 \pm 13.57$ for CD24) and $\mathrm{p}$ value was $<0.05$ as summarized in Table 3 . A significant difference was observed in expression of all three biomarkers among patients in whom clinical response were complete, partial and non-responders $(12.67 \pm 4.31$ vs. $21.41 \pm 9.38$ vs. $39.01 \pm 14.74$ for $\operatorname{Oct} 4, \mathrm{p}<0.0001$; $11.57 \pm 5.93$ vs. $20.98 \pm 10.13$ vs. $38.83 \pm 16.35$ for Nanog, $\mathrm{p}<0.0001 ; 16.76 \pm 9.54$ vs. $29.93 \pm 14.02$ vs. $48.81 \pm 15.34$ for $\mathrm{CD} 24, \mathrm{p}<0.0001)$.

Survival analysis: Recurrence-free survival was $71 \%$ at 3 years. In Cox regression analysis, advanced ypTNM stage $(\mathrm{P}=0.03)$, clinical non-response $(\mathrm{p}=0.006)$, pathologic non-response $(\mathrm{P}<0.002)$, positive expression of all three biomarkers (dichotomized variable, $p=0.0001$ ) and high expression of all three biomarkers (continuous variable, $p=0.0001$ ) had a significant negative effect on recurrence-free survival as shown in Table 4 . The overall survival rate of all 50 patients was $66 \%$ at 3 years. Cox regression analyses showed that advanced
Table 1. Demographic and Clinico-Pathological Characteristics of Patients

\begin{tabular}{|c|c|}
\hline Characteristics & Patient $n=50(\%)$ \\
\hline \multicolumn{2}{|l|}{ Age } \\
\hline $25-50 \mathrm{yrs}$ & $30(60)$ \\
\hline$>50 \mathrm{yrs}$ & $20(40)$ \\
\hline \multicolumn{2}{|l|}{ Sex } \\
\hline Male & $41(82)$ \\
\hline Female & $09(18)$ \\
\hline \multicolumn{2}{|l|}{ Tobacco consumption } \\
\hline Chewing & $41(82)$ \\
\hline Smoking & $21(42)$ \\
\hline Betel nut chewing & $30(60)$ \\
\hline Alcohol intake & $13(26)$ \\
\hline \multicolumn{2}{|l|}{ Site of primary tumour } \\
\hline Buccal Mucosa & $21(42)$ \\
\hline Tongue & $14(28)$ \\
\hline Retro moral trigone & $05(10)$ \\
\hline Cheek & $04(8)$ \\
\hline Palate & $03(6)$ \\
\hline Lip & $03(6)$ \\
\hline \multicolumn{2}{|l|}{ Histological grading } \\
\hline Well Differentiated & $09(18)$ \\
\hline Moderately differentiated & $36(72)$ \\
\hline Poorly differentiated & $05(10)$ \\
\hline \multicolumn{2}{|l|}{ Clinical N stage } \\
\hline No & $11(22)$ \\
\hline $\mathrm{N}+$ & $39(78)$ \\
\hline \multicolumn{2}{|l|}{ Clinical TNM stage } \\
\hline Stage III & $22(44)$ \\
\hline Stage IVa/b & $28(56)$ \\
\hline \multicolumn{2}{|l|}{ ypN stage* } \\
\hline 0 & $35(70)$ \\
\hline 1 & $12(24)$ \\
\hline 2 & $03(6)$ \\
\hline \multicolumn{2}{|l|}{ Yp TNM stage $* *$} \\
\hline 0 & $20(40)$ \\
\hline 1 & $09(18)$ \\
\hline 2 & $06(12)$ \\
\hline 3 & $08(16)$ \\
\hline 4 & $07(14)$ \\
\hline \multicolumn{2}{|l|}{ Clinical response } \\
\hline Complete Responder & $15(30)$ \\
\hline Partial Responder & $23(46)$ \\
\hline Stable disease & $07(14)$ \\
\hline Progressive disease & $05(10)$ \\
\hline \multicolumn{2}{|l|}{ Pathological response } \\
\hline RG1 & $15(30)$ \\
\hline RG2 & $22(44)$ \\
\hline RG3 & 07 (14) \\
\hline RG4 & $06(12)$ \\
\hline
\end{tabular}

*ypN stage, pathologic N stage after neoadjuvant therapy; ** ypTNM stage, pathologic TNM stage after neoadjuvant therapy 
Table 2. The Association of Clinical and Pathological Response after Neoadjuvant Therapy with Expression of the Biomarkers and Clinic-Pathological Parameters

\begin{tabular}{|c|c|c|c|c|c|c|c|c|}
\hline \multirow[b]{2}{*}{ Parameters } & \multirow[t]{2}{*}{ No. of cases $(\%)$} & \multicolumn{3}{|c|}{ Clinical Response } & \multirow[b]{2}{*}{ p-value } & \multicolumn{3}{|c|}{ Pathological Response } \\
\hline & & $\begin{array}{l}\text { Complete } \\
\text { response }\end{array}$ & $\begin{array}{c}\text { Partial } \\
\text { response }\end{array}$ & $\begin{array}{l}\text { No } \\
\text { response }\end{array}$ & & Response & $\begin{array}{l}\text { No } \\
\text { response }\end{array}$ & p-value \\
\hline \multicolumn{9}{|l|}{ Oct4 Expression } \\
\hline Positive & $23(46)$ & 5 & 7 & 11 & 0.001 & 12 & 11 & 0.003 \\
\hline Negative & $27(54)$ & 10 & 16 & 1 & & 25 & 2 & \\
\hline \multicolumn{9}{|c|}{ Nanog Expression } \\
\hline Positive & $27(54)$ & 2 & 15 & 10 & 0.0001 & 16 & 11 & 0.02 \\
\hline Negative & $23(46)$ & 13 & 8 & 2 & & 21 & 2 & \\
\hline \multicolumn{9}{|c|}{$C D 24$ Expression } \\
\hline Positive & $29(58)$ & 2 & 16 & 11 & 0.01 & 17 & 12 & 0.03 \\
\hline Negative & $21(42)$ & 12 & 7 & 2 & & 20 & 1 & \\
\hline \multicolumn{9}{|l|}{ Age: } \\
\hline $25-50 \mathrm{yrs}$ & $30(60)$ & 10 & 15 & 5 & 0.33 & 23 & 7 & 0.84 \\
\hline$>50 \mathrm{yrs}$ & $20(40)$ & 5 & 8 & 7 & & 14 & 6 & \\
\hline \multicolumn{9}{|l|}{ Sex } \\
\hline Male & $41(82)$ & 11 & 20 & 10 & 0.56 & 31 & 10 & 0.89 \\
\hline Female & 09 (18) & 4 & 3 & 2 & & 6 & 3 & \\
\hline \multicolumn{9}{|l|}{ Grade } \\
\hline WD & 09 (18) & 3 & 4 & 2 & & 7 & 2 & \\
\hline MD & $36(72)$ & 10 & 17 & 9 & 0.98 & 26 & 10 & 0.9 \\
\hline PD & $05(10)$ & 2 & 2 & 1 & & 4 & 1 & \\
\hline \multicolumn{9}{|l|}{ Clinical N stage } \\
\hline No & $11(22)$ & 4 & 5 & 2 & 0.83 & 7 & 4 & 0.61 \\
\hline $\mathrm{N}+$ & $39(78)$ & 11 & 18 & 10 & & 30 & 9 & \\
\hline \multicolumn{9}{|l|}{$\begin{array}{l}\text { Clinical TNM } \\
\text { stage }\end{array}$} \\
\hline Stage III & $22(44)$ & 6 & 12 & 4 & 0.52 & 16 & 6 & 0.82 \\
\hline Stage $1 \mathrm{Va} / \mathrm{b}$ & $28(56)$ & 9 & 11 & 8 & & 21 & 7 & \\
\hline
\end{tabular}

ypN stage $(\mathrm{P}=0.02)$, advanced ypTNM stage $(\mathrm{P}=0.01)$, clinical non-response $(\mathrm{p}=0.04)$, pathologic non-response $(\mathrm{P}=0.008)$, positive expression of all three biomarkers (dichotomized variable, $\mathrm{p}=0.0001$ ) and high expression of all three biomarkers (continuous variable, $p=0.0001$ ) were significantly associated with decreased overall survival.

Survival rates were compared between clinical outcome of neoadjuvant chemoradiation and expression of CSC markers using Kaplan-Meier method. Patients with overexpression of markers had a significantly unfavorable outcome compared to those with negative expression (Log-rank test, $\mathrm{p}<0.05$ ) (Figure 2). The cumulative survival rate for 3 years in the positive expression group was 59\% (Oct4), 62\% (Nanog) and 60\%

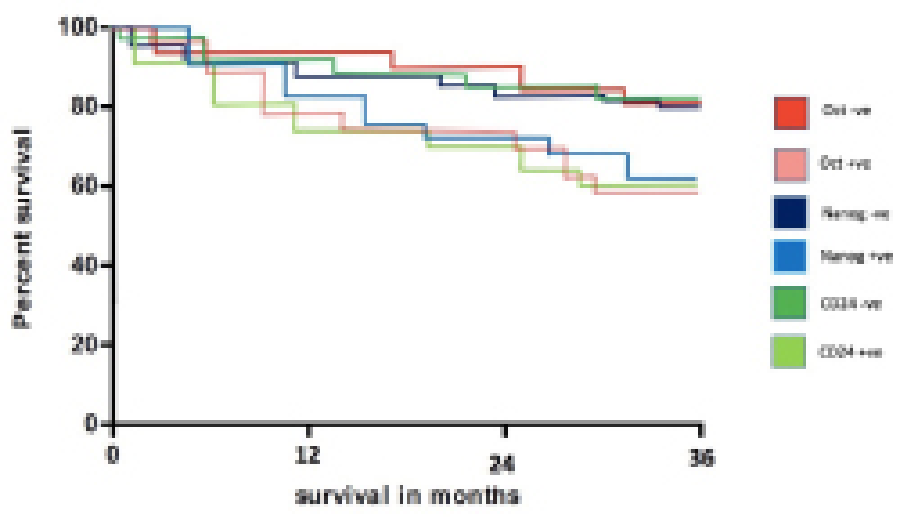

Figure 2. Kaplan Meier Graphs Showing the Cumulative 3-Year Survival of Patients According to Immunoreactivity for Oct 4 , Nanog and CD24 which was $59 \%$ for Oct $4+v e, 62 \%$ for Nanog +ve and $60 \%$ for CD24 +ve as compared to $81.0 \%$ for Oct4 -ve, $79 \%$ for Nanog -ve and $82 \%$ for CD24 -ve respectively (Log-rank test, $\mathrm{P}<0.05$ ). 
Table 3. The Association of Expression of Biomarkers with Demographic and Response to Neo-Adjuvant Therapy

\begin{tabular}{|c|c|c|c|c|c|c|}
\hline Parameter & $\begin{array}{l}\text { Oct-4 Expression } \\
(\text { Mean } \pm \mathrm{SD})\end{array}$ & $\mathrm{p}$ value & $\begin{array}{l}\text { Nanog Expression } \\
\quad(\text { Mean } \pm \text { SD) }\end{array}$ & P-value & $\begin{array}{c}C D 24 \text { Expression } \\
(\text { Mean } \pm \mathrm{SD})\end{array}$ & P-value \\
\hline \multicolumn{7}{|l|}{ Age } \\
\hline $25-50 \mathrm{yrs}$ & $28.48 \pm 12.15$ & 0.125 & $27.22 \pm 11.96$ & 0.494 & $41.37 \pm 13.81$ & 0.665 \\
\hline$>50 \mathrm{yrs}$ & $23.30 \pm 10.43$ & & $25.02 \pm 9.54$ & & $39.70 \pm 12.42$ & \\
\hline \multicolumn{7}{|l|}{ Sex } \\
\hline Male & $26.52 \pm 13.73$ & 0.851 & $26.71 \pm 12.48$ & 0.6003 & $38.44 \pm 14.80$ & 0.918 \\
\hline Female & $25.55 \pm 15.45$ & & $24.34 \pm 10.73$ & & $37.89 \pm 12.67$ & \\
\hline \multicolumn{7}{|l|}{ Histological Grade } \\
\hline WD & $25.10 \pm 11.26$ & & $24.20 \pm 11.32$ & & $38.94 \pm 12.10$ & \\
\hline MD & $28.75 \pm 12.98$ & 0.731 & $23.16 \pm 13.54$ & 0.846 & $37.50 \pm 11.61$ & 0.813 \\
\hline $\mathrm{PD}$ & $27.16 \pm 10.89$ & & $26.75 \pm 14.46$ & & $40.81 \pm 10.43$ & \\
\hline \multicolumn{7}{|l|}{ Clinical N Stage } \\
\hline N0 & $23.41 \pm 11.17$ & 0.835 & $28.61 \pm 13.40$ & 0.521 & $37.51 \pm 11.75$ & 0.629 \\
\hline $\mathrm{N}$ & $22.52 \pm 12.83$ & & $25.93 \pm 11.79$ & & $35.73 \pm 10.46$ & \\
\hline \multicolumn{7}{|l|}{ Clinical TNM Stage } \\
\hline III & $26.29 \pm 12.14$ & 0.881 & $26.68 \pm 12.74$ & 0.851 & $37.45 \pm 14.47$ & 0.841 \\
\hline IV & $25.74 \pm 13.43$ & & $27.35 \pm 12.35$ & & $36.68 \pm 12.64$ & \\
\hline \multicolumn{7}{|l|}{ Clinical Response: } \\
\hline Complete Responders & $12.67 \pm 4.31$ & $<0.0001$ & $11.57 \pm 5.93$ & $<0.0001$ & $16.76 \pm 9.54$ & $<0.0001$ \\
\hline Partial Responders & $21.41 \pm 9.38$ & & $20.98 \pm 10.13$ & & $29.93 \pm 14.02$ & \\
\hline Non Responders & $39.01 \pm 14.74$ & & $38.83 \pm 16.35$ & & $48.81 \pm 15.34$ & \\
\hline \multicolumn{7}{|l|}{ Histological Response } \\
\hline Response (R1/R2) & $17.32 \pm 6.83$ & 0.0001 & $18.86 \pm 10.21$ & 0.0001 & $22.37 \pm 12.26$ & 0.0001 \\
\hline No response (R3/R4) & $37.15 \pm 13.65$ & & $39.92 \pm 13.93$ & & $47.93 \pm 13.57$ & \\
\hline
\end{tabular}

Table 4. Cox Regression Analyses of Recurrence-Free Survival and Overall Survival

\begin{tabular}{|c|c|c|c|c|c|c|}
\hline & \multicolumn{3}{|c|}{ Recurrence-free survival } & \multicolumn{3}{|c|}{ Overall survival } \\
\hline & Hazard ratio & $95 \% \mathrm{CI}$ & $\mathrm{p}$ value & Hazard ratio & $95 \% \mathrm{CI}$ & P-value \\
\hline Age & 1.18 & $0.63-3.21$ & 0.87 & 1.43 & $0.82-3.14$ & 0.32 \\
\hline Sex & 0.92 & $0.56-1.67$ & 0.65 & 0.61 & $0.35-1.39$ & 0.78 \\
\hline Tobacco consumption & 1.08 & $0.73-1.75$ & 0.76 & 1.12 & $0.72-1.84$ & 0.63 \\
\hline Betel nut chewing & 0.74 & $0.41-1.23$ & 0.91 & 0.89 & $0.46-1.73$ & 0.88 \\
\hline Alcohol intake & 0.87 & $0.57-1.34$ & 0.67 & 0.76 & $0.49-1.82$ & 0.61 \\
\hline Site of primary tumour & 0.67 & $0.39-1.42$ & 0.29 & 0.72 & $0.35-1.57$ & 0.58 \\
\hline Histological grading & 1.52 & $0.77-2.31$ & 0.76 & 1.41 & $0.68-2.78$ & 0.49 \\
\hline Clinical N stage & 1.74 & $0.41-2.57$ & 0.42 & 1.53 & $0.72-2.41$ & 0.16 \\
\hline Clinical TNM stage & 1.27 & $0.32-2.62$ & 0.32 & 1.47 & $0.58-2.94$ & 0.37 \\
\hline ypN stage ${ }^{a}$ & 1.24 & $0.43-2.47$ & 0.13 & 1.76 & $1.23-2.35$ & 0.02 \\
\hline yp TNM stage ${ }^{b}$ & 1.42 & $1.12-2.53$ & 0.03 & 1.76 & $1.49-2.83$ & 0.01 \\
\hline Clinical response ${ }^{c}$ & 1.48 & $1.32-3.63$ & 0.006 & 1.38 & $1.18-3.27$ & 0.04 \\
\hline Pathological response ${ }^{\mathrm{c}}$ & 1.52 & $1.24-3.11$ & 0.002 & 1.46 & $1.02-3.71$ & 0.008 \\
\hline Oct4 positive $(>10 \%)$ & 1.42 & $1.89-2.61$ & 0.001 & 1.75 & $1.37-2.56$ & 0.001 \\
\hline Oct $4^{\mathrm{d}}$ & 1.56 & $1.24-2.51$ & 0.001 & 1.17 & $1.03-1.67$ & 0.001 \\
\hline Nanog positive $(>10 \%)$ & 1.56 & $1.18-2.47$ & 0.001 & 1.59 & $1.24-2.32$ & 0.001 \\
\hline Nanog ${ }^{d}$ & 1.11 & $1.79-2.81$ & 0.001 & 1.71 & $1.12-2.39$ & 0.001 \\
\hline CD 24 positive $(>10 \%)$ & 1.75 & $1.03-2.44$ & 0.001 & 1.72 & $1.23-2.57$ & 0.001 \\
\hline $\mathrm{CD} 24^{\mathrm{d}}$ & 1.82 & $1.52-2.48$ & 0.001 & 1.49 & $1.12-2.55$ & 0.001 \\
\hline
\end{tabular}

${ }^{a} \mathrm{ypN}$ stage, pathologic $\mathrm{N}$ stage after neoadjuvant therapy; ${ }^{\mathrm{b} y p T N M}$ stage, pathologic TNM stage after neoadjuvant therapy; ${ }^{\mathrm{c} C l i n i c a l}$ and pathologic response was evaluated as described in Materials and methods; ${ }^{\mathrm{d} C o n t i n u o u s ~ v a r i a b l e . ~}$ 
(CD24) whereas that in the negative expression groups was $81 \%$ (Oct4), 79\% (Nanog) and 82\% (CD24).

\section{Discussion}

Cancer stem cells (CSCs) play an important role in initiation, propagation, metastasis, recurrence, and therapeutic failure of OSCC. The CSC theory hypothesises that unregulated asymmetric division of CSCs generate dissimilar population of differentiated progenitor cells that eventually make up a heterogeneous tumor (Sharpless and DePinho 2007; Gil et al., 2008). CSCs are believed to be highly tumorigenic and potentially metastatic, with resistance to most forms of radiation and chemotherapy (Nichols et al., 1998). Tumor growth is targeted by inhibiting DNA synthesis or cell division using anti-cancer drugs. Due to high clonogenic and tumorigenic capacity, some slow dividing CSCs protect themselves from therapy and lead to resistance (Yanamoto et al., 2014; Gil et al., 2008). Therefore failure of cancer treatment may be explained by improved understanding of the biological characteristics of CSCs. Identification of these CSCs markers may help in predicting therapeutic response and serve to optimize treatment plan to improve survival.

Embryological stem cells have a central regulatory network that involves three master regulators for maintenance of the undifferentiated state. These include Oct4 (Pit Oct Unc [POU] domain transcription factor), Nanog (homeodomain transcription factor), and Sox-2 (high mobility group protein) (Nichols et al., 1998; Pesce et al., 1998; Mitsui et al., 2003). Additionally, Oct-4 and Nanog are purposed to be two of the four major factors that allow reprogramming of differentiated cells into pluripotent cells. Abnormal expression of these factors in stem cell and tumor tissues might play a vital role in tumor transformation, tumorigenicity, and tumor metastasis (Nichols et al., 1998; Pesce et al., 1998; Mitsui et al., 2003).

CD24 is 27-amino-acid single-chain protein that is heavily O-and N-glycosylated glycosylphosphatidylinositol (GPI) - linked cell surface protein (Koukourakis et al., 2012; Kristiansen et al., 2003a). CD24 is a B-cell specific marker expressed in the early stages of B-cell development and also expressed in developing or regenerating tissue (Kristiansen et al., 2003a; Choi et al., 2007). P- Selectin is its only ligand identified till date (Koukourakis et al., 2012; Sung et al., 2010; Chou et al., 2007). CD24 functions as ligand to P-selectin, by virtue of which it facilitates interaction with platelets or endothelial cells, thereby increasing metastatic potential of tumour. CD24 expression has been identified as a prognostic marker in variety of tumors (Kristiansen et al., 2003a; 2003b; Sung et al., 2010; Choi et al., 2007; Chou et al., 2007; Sano et al., 2009)

Tsai et al showed increased expression of Oct4 and Nanog correlated with a cisplatin-resistant phenotype as well as cancer recurrence while negatively correlated with differentiation status in OSCC (Tsai et al., 2011). In a study of Siu et al showed that embryonic stem cells express Oct4, which is lost upon differentiation. It was expressed in most invasive oral cancer cell lines indicating that Oct4 is a marker of invasiveness (Siu et al., 2012). Chiou et al., (2008) demonstrated that OSCC cases with expression of Oct-4, Nanog, and CD133 had worst survival. It has also been shown that overexpression of Oct-4 and Nanog positively correlates with stage and chemo resistance, while negatively correlates with tumour grade. Habu et al., (2015) evaluated expression of Oct4 and Nanog in 50 patients of HNSCC and suggested that these CSCs contribute significantly to the development of delayed neck metastasis by enhancing cell motility and invasiveness. In adenocarcinoma of lung, Oct4 and Nanog overexpression was associated with higher stage and shorter survival (Chiou et al., 2010). Hence, Oct-4 and Nanog may acts as useful prognostic biomarkers for OSCC.

Koukourakis et al., (2012) evaluated the role of CD24 and Oct4 in 74 locally advanced HNSCC and reported that extensive presence of Oct4 and CD24 was directly linked with increased proliferation index and poor prognosis. Kwon et al., (2007) analyzed the expression of CD24 in 73 cases of uterine cervical SCC and found patients with negative CD24 having 20\% less total failure and distant metastatic rates as compared to CD24 positive patients. The 5 -year distant metastasis-free survival rate of CD24-negative patients was significantly greater than that of the CD24-positive patients $(84.7 \%$ vs. $66.7 \%$, respectively, $\mathrm{p}=0.0497)$. Kristiansen et al., (2003a) evaluated CD24 protein expression by immunohistochemistry in ovarian cancer and suggested a highly significant association of CD24 overexpression with shortened patient survival. In addition, Kristiansen et al., (2003a) reported that CD24 expression was associated with shortened disease free survival in breast cancer patients. Sung et al., (2010) evaluated the CD24 expression of 140 patients with cervical SCC treated with chemo-radiotherapy after radical hysterectomy and concluded that CD24 expression was significantly associated with loco regional failure-free survival, distant metastasis-free survival and overall survival. Choi et al., (2007) reported that a CD24 overexpression and loss of apical localization strongly predicts high tumor grade and stromal invasion in patients of urethral carcinoma.

We have also observed statistically significant association between negative expression $(<10 \%)$ of Oct4, Nanog and CD24 and clinical or pathological response, there by indicating the possible potential of these markers as predictors for evaluating response to neoadjuvant chemo-radiotherapy in locally advanced OSCC.

We further observed that in advanced ypN and ypTNM stage, overexpression of Oct4, Nanog and CD24 have a significant negative impact on survival outcomes. Moreover, patients exhibiting complete and partial response (clinically) or complete (RG1) and near-complete response (RG2) to neoadjuvant therapy show improved overall survival and recurrence free survival as compared to those with non-responders (clinically) or patients with residual tumor (RG3/RG4 pathologically) respectively. These findings are supported by previous reports (Driemel et al., 2009). Nevertheless, our study has few limitations. The sample size of study is small and the study design is retrospective. This may have introduced a selection bias. 
However, our inclusion criteria were stringent and our study protocol was strictly followed.

Currently, no single biomarker has been approved to accurately define CSCs in OSCC. However, a set of markers may help to target CSC population and identify patients with poor prognosis. This study may open new avenues for in-depth analysis and validation of the stem cell related genes studied herein as a useful predictive markers of response to the neoadjuvant therapy in OSCC patients. Further studies may be planned to focus to detail altered molecular pathways involved in resistance. The expression of these markers needs prospective validation to further elucidate their role as a predictive biomarkers for chemo-radiation response.

In conclusion, our results suggest that expression of biomarkers Oct4, Nanog and CD24 have a significant impact on treatment response and survival in patients with locally advanced OSCC treated with neoadjuvant chemo-radiation. Survival of these patients is significantly affected by ypN stage, ypTNM stage, expression of all three biomarkers, clinical and pathological response to neoadjuvant therapy.

\section{Funding}

The study was partially funded by intramural research grant no, Dr. Ram Manohar Lohia Institute of Medical Sciences, Lucknow-226010, Uttar Pradesh, India.

\section{Conflict of interest}

All authors have stated explicitly that there is no conflict of interest in connection with this article.

\section{References}

Amin MB, Greene FL, Edge SB, et al (2017). The eighth edition AJCC cancer staging manual: continuing to build a bridge from a population-based to a more "personalized" approach to cancer staging. CA Cancer J Clin, 67, 93-9.

Braun OM, Neumeister B, Popp W, et al (1989). Histologic tumor regression grades in squamous cell carcinoma of the head and neck after preoperative radiochemotherapy. Cancer, 63, 1097-1100.

Chiou SH, Wang ML, Chou YT, et al (2010). Co-expression of Oct4 and Nanog enhances malignancy in lung adenocarcinoma by inducing cancer stem cell-like properties and epithelial-mesenchymal transdifferentiation. Cancer Res, 70, 10433-4.

Chiou SH, Yu CC, Huang CY, et al (2008). Positive correlations of Oct-4 and Nanog in oral cancer stem-like cells and high-grade oral squamous cell carcinoma. Clin. Cancer Res, 14, 4085-4095.

Choi YL, Lee SH, Kwon GY, et al (2007). Overexpression of CD24: association with invasiveness in urothelial carcinoma of the bladder. Arch Pathol Lab Med, 131, 275-81.

Chou YY, Jeng YM, Lee TT, et al (2007). Cytoplasmic CD24 expression is a novel prognostic factor in diffuse-type gastric adenocarcinoma. Ann Surg Oncol, 14, 2748-58.

Driemel O, Ettl T, Kölbl O, et al (2009). Outcome and histopathologic regression in oral squamous cell carcinoma after preoperative radiochemotherapy. Strahlenther Onkol, 185, 296-302.

Gil J, Stembalska A, Pesz KA, et al (2008). Cancer stem cells: the theory and perspectives in cancer therapy. J Appl Genet, 49, 193-9.
Guo Z, Li LQ, Jiang JH, et al (2014). Cancer stem cell markers correlate with early recurrence and survival in hepatocellular carcinoma. World J Gastroenterol, 20, 2098.

Gupta GP, Massagué J (2006). Cancer metastasis: building a framework. Cell, 127, 679-95.

Gupta T, Agarwal JP, Ghosh-Laskar S, et al (2009). Radical radiotherapy with concurrent weekly cisplatin in loco-regionally advanced squamous cell carcinoma of the head and neck: a single-institution experience. Head Neck Oncol, 1, 17.

Habu N, Imanishi Y, Kameyama K, et al (2015). Expression of Oct3/4 and Nanog in the head and neck squamous carcinoma cells and its clinical implications for delayed neck metastasis in stage I/II oral tongue squamous cell carcinoma. $B M C$ Cancer, 15, 730.

Jemal A, Bray F, Center MM, et al (2011). Global cancer statistics. CA Cancer J Clin, 61, 69-90.

Kirita T, Yamanaka Y, Imai Y, et al (2012). Preoperative concurrent chemo radiotherapy for stages II-IV oral squamous cell carcinoma: A retrospective analysis and the future possibility of this treatment strategy. Int J Oral Maxillofac Surg, 41, 421-8.

Koukourakis MI, Giatromanolaki A, Tsakmaki V, Danielidis V, Sivridis E (2012). Cancer stem cell phenotype relates to radio-chemotherapy outcome in locally advanced squamous cell head-neck cancer. Br J Cancer, 106, 846.

Kristiansen G, Schlüns K, Yongwei Y, et al (2003b). CD24 is an independent prognostic marker of survival in nonsmall cell lung cancer patients. Br J Cancer, 88, 231.

Kristiansen G, Winzer KJ, Mayordomo E, et al (2003a). CD24 expression is a new prognostic marker in breast cancer. Clin Cancer Res, 9, 4906-13.

Kwon GY, Ha H, Ahn G, et al (2007). Role of CD24 protein in predicting metastatic potential of uterine cervical squamous cell carcinoma in patients treated with radiotherapy. Int $J$ Radiat Oncol Biol Phys, 69, 1150-6.

Mitsui K, Tokuzawa Y, Itoh H, et al (2003). The homeoprotein Nanog is required for maintenance of pluripotency in mouse epiblast and ES cells. Cell, 113, 631-42.

Nichols J, Zevnik B, Anastassiadis K, et al (1998). Formation of pluripotent stem cells in the mammalian embryo depends on the POU transcription factor Oct4. Cell, 95, 379-91.

Pesce M, Wang X, Wolgemuth DJ, Schöler HR (1998). Differential expression of the Oct-4 transcription factor during mouse germ cell differentiation. Mech Dev, 71, 89-98.

Pindborg JJ, Reichart PA, Smith CJ, Van der Waal I (2012). Histological Typing of Cancer and Precancer of the Oral Mucosa: In Collaboration with LH Sobin and Pathologists in 9 Countries. Springer Science and Business Media.

Robbins KT, Clayman G, Levine PA, et al (2002). Neck dissection classification update: revisions proposed by the American Head and Neck Society and the American Academy of Otolaryngology-Head and Neck Surgery. Arch Otolaryngol Head Neck Surg, 128, 751-8.

Sano A, Kato H, Sakurai S, et al (2009). CD24 expression is a novel prognostic factor in esophageal squamous cell carcinoma. Ann Surg Oncol, 16, 506-14.

Sharpless NE, DePinho RA (2007). How stem cells age and why this makes us grow old. Nat Rev Mol, 8, 703.

Siu A, Lee C, Dang D, Lee C, Ramos DM (2012). Stem cell markers as predictors of oral cancer invasion. Anticancer Res, 32, 1163-6.

Soni P, Husain N, Awasthi R, et al (2014). Revisiting epidermal growth factor receptor in glioblastoma multiforme: Does it play a role in response to therapy?. Indian J Pathol Microbiol, 57, 390.

Sung CO, Park W, Choi YL, et al (2010). Prognostic significance 
of CD24 protein expression in patients treated with adjuvant radiotherapy after radical hysterectomy for cervical squamous cell carcinoma. Radiat Oncol, 95, 359-64.

Tsai LL, Yu CC, Chang YC, et al (2011). Markedly increased Oct4 and Nanog expression correlates with cisplatin resistance in oral squamous cell carcinoma. J Oral Pathol Med, 40, 621-8.

Yanamoto S, Yamada SI, Takahashi H, et al (2014). Expression of the cancer stem cell markers CD44v6 and ABCG2 in tongue cancer: effect of neoadjuvant chemotherapy on local recurrence. Int J Oncol, 44, 1153-62.

\section{(c) (1) 8)}

This work is licensed under a Creative Commons AttributionNon Commercial 4.0 International License. 